\title{
Erratum to: A case of rapid conversion to psychosis of delusional misidentification associated with derealisation, verbal memory impairment and FDG-PET imaging abnormalities
}

\author{
Anna Comparelli • Georgios D. Kotzalidis • \\ Simone Di Pietro · Antonio Del Casale • \\ Antonella De Carolis
}

Published online: 14 June 2013

(c) Springer-Verlag Berlin Heidelberg 2013

\section{Erratum to: Eur Child Adolesc Psychiatry}

DOI 10.1007/s00787-013-0420-z

In the original publication, the family name of fourth author was erroneously reported as "Casale". The correct family name should be "Del Casale".

The online version of the original article can be found under doi:10.1007/s00787-013-0420-z.
A. Comparelli $(\varangle) \cdot$ G. D. Kotzalidis · S. Di Pietro ·
A. Del Casale
Unit of Psychiatry, NESMOS (Neurosciences, Mental Health and Sensory Organs) Department, School of Medicine and Psychology, Sant'Andrea Hospital, Sapienza University of Rome, Via di Grottarossa 1035-39, 00189 Rome, Italy e-mail: anna.comparelli@uniroma1.it

\section{A. Comparelli - A. De Carolis}

Unit of Nuclear Medicine, School of Medicine e Psychology,

Sant'Andrea Hospital, Sapienza University of Rome,

Rome, Italy

\section{A. De Carolis}

Unit of Psychological Medicine, NESMOS (Neurosciences,

Mental Health and Sensory Organs) Department, School

of Medicine and Psychology, Sant'Andrea Hospital,

Sapienza University of Rome, Rome, Italy 